\title{
CHARACTERISTICS OF GENTAMICIN UPTAKE IN THE ISOLATED CRISTA AMPULLARIS OF THE INNER EAR OF THE GUINEA PIG
}

\author{
Sally E. Williams, ${ }^{*}$ David E. Smith $\dagger$ and Jochen Schacht ${ }^{*} \ddagger$ \\ * Kresge Hearing Research Institute, and † College of Pharmacy, The University of Michigan, Ann \\ Arbor, MI 48109, U.S.A.
}

(Received 27 February 1986; accepted 22 May 1986)

\begin{abstract}
The characteristics of aminoglycoside uptake in the inner ear were investigated in the isolated crista ampullaris. The organ was incubated with radiolabeled gentamicin, and the stable, nonexchangeable radioactivity was considered the intracellular pool of the drug. Gentamicin was transported against a concentration gradient, and the resulting tissue to medium ratios ranged from 1.4 to 18.6. Transport was inhibited by reduction in temperature or by the addition of metabolic blockers, indicative of an energy-dependent component. The uptake system showed at least two sites, the first a high-affinity site with a dissociation constant $K_{D}=39 \mathrm{nM}$ and a capacity of $n=0.2$ pmole/crista; the second had a $K_{D}=16 \mu \mathrm{M}$ and a capacity of $n=11$ pmoles/crista. Aminoglycosides competed with uptake of gentamicin in the order netilmicin $\geq$ neomycin $>$ tobramycin, and polyamines competed in the order spermine $>$ spermidine $>$ putrescine; glucosamine and the basic amino acids lysine and asparagine were ineffective, excluding the participation of glucose and basic amino acid transport systems in gentamicin uptake. These results along with direct comparisons with some characteristics of putrescine uptake suggest that aminoglycoside and polyamine transport have common features in the crista ampullaris.
\end{abstract}

The existence of an active cellular uptake mechanism for aminoglycoside antibiotics has been well established in bacteria and in the kidney. Bacterial uptake of aminoglycosides proceeds in two energy-dependent phases, is susceptible to competition by amino acids and divalent cations, and is inhibited by blockers of electron transport and uncouplers of the protonmotive force [1]. Renal drug uptake has also been described in some detail and is known to be energy dependent [2-5].

Evidence has been presented only recently that the inner ear may also possess an active transport system for aminoglycosides. The pharmacokinetics of gentamicin in the inner ear [6] indicated that the drug occupies two distinctly different binding sites in the course of its action. Electrophysiological studies of its cochlear toxicity [7] suggested the presence of an extracellular and an intracellular compartment. In addition, it was shown that an energy-dependent step is required for the expression of gentamicin ototoxicity [8]. More recently, Schacht and Van De Water directly demonstrated the uptake and accumulation of gentamicin in the developing inner ear in culture [9].

While this evidence strongly supports an active transport of aminoglycosides in the inner ear, its mechanisms remain to be elucidated. The aim of this study was to examine and characterize the aminoglycoside uptake system in the inner ear. The crista ampullaris of the guinea pig was chosen since this vestibular structure is susceptible to damage by aminoglycosides and is easily and consistently dissected. In addition, an earlier study of gentamicin

\$ To whom correspondence should be addressed. binding had shown the usefulness of this preparation for reproducible in vitro incubations [10].

\section{MATERIALS AND METHODS}

Dissection. Cristae ampullares were dissected from pigmented guinea pigs (180-240 g; Murphy Breeding Laboratories, Plainsfield, IN). After decapitation the temporal bones were separated into the cochlear and vestibular parts and placed in Hanks' balanced salt solution with an osmolarity of $300 \mathrm{mOsM}$ (Gibco Co., Grand Island, NY) at room temperature. The cristae were removed intact and the ampullae were opened with microscissors to permit tree diffusion of the medium.

Uptake studies. Isolated cristae were incubated individually in polypropylene tubes (because of the affinity of aminoglycosides and polyamines for glass surfaces) at $37^{\circ}$ in a final volume of $0.20 \mathrm{ml}$ of Hanks' solution $\left(136.9 \mathrm{mM} \mathrm{NaCl}, 4.16 \mathrm{mM} \mathrm{NaHCO}_{3}\right.$, $0.33 \mathrm{mM} \quad \mathrm{Na}_{2} \mathrm{HPO}_{4}, \quad 5.37 \mathrm{mM} \mathrm{KCl}, \quad 0.44 \mathrm{mM}$ $\mathrm{KH}_{2} \mathrm{PO}_{4}, 0.49 \mathrm{mM} \mathrm{MgCl}, 1.26 \mathrm{mM} \mathrm{CaCl}_{2}, 5.5 \mathrm{mM}$ glucose; $\mathrm{pH}, 7.4)$. The additional assay components and some details of the incubation varied with the individual experiments as described below. The following reagents were added: for gentamicin uptake, $0.5 \mu \mathrm{Ci}{ }^{125} \mathrm{I}$-labeled gentamicin $(19.9 \mathrm{Ci} / \mathrm{mmole}$; American Bioclinical, Portland, OR) and unlabeled gentamicin to a final concentration of $1 \mu \mathrm{M}$, except as noted in the gentamicin dose-response study; for putrescine uptake, $0.1 \mu \mathrm{Ci} \quad\left[1,4-{ }^{14} \mathrm{C}\right]$ putrescine dihydrochloride $\quad(109 \mathrm{mCi} / \mathrm{mmole}$ Amersham Corp., Arlington Heights, IL); (final concentration, $4.6 \mu \mathrm{M}$ ); for $\gamma$-aminobutyric acid (GABA) uptake, $0.008 \mu \mathrm{Ci} \gamma-\left[2,3-{ }^{3} \mathrm{H}(\mathrm{N})\right]$-aminobutyric acid $(98.5 \mathrm{Ci} /$ 
mmole; New England Nuclear, Boston, MA) and unlabeled GABA to a final concentration of $1 \mu \mathrm{M}$; for mannitol uptake, $0.135 \mu \mathrm{Ci} \mathrm{D}-\left[1-{ }^{3} \mathrm{H}(\mathrm{N})\right]-$ mannitol (17 Ci/mmole; New England Nuclear); and for butanol uptake, $0.17 \mu \mathrm{Ci}\left[{ }^{14} \mathrm{C}\right.$ ]butanol (gift from $\mathrm{Dr}$. $\mathrm{N}$. Weiner, The University of Michigan).

The reactions were started by the addition of a crista to the incubation mixture except for experiments in which inhibitors or competitors were tested. In these cases, each crista was preincubated for $5 \mathrm{~min}$ in the presence of the antagonist, and the incubation was started by the addition of the radiolabeled compound.

At the end of the incubation period, the radioactive medium was replaced by $1 \mathrm{ml}$ of medium containing $1 \mathrm{mM}$ unlabeled compound. Five minutes thereafter the crista was separated on a Millipore RA filter (Millipore Corp., Bedford, MA) and rinsed with $10 \mathrm{ml}$ of Ringer's solution. The filter area containing the crista was cut out, dissolved in $50 \mu \mathrm{l}$ of $1 \mathrm{~N} \mathrm{NaOH}\left(30 \mathrm{~min}\right.$ at $\left.80^{\circ}\right)$ and neutralized with $150 \mathrm{ml}$ acetic acid (glacial acetic acid in $\mathrm{H}_{2} \mathrm{O}, 1: 40$ ). Radioactivity was determined by liquid scintillation counting. A filter area of the same size adjacent to the crista was used as a correction for radioactivity bound to the filter.

The incubations with labeled butanol were terminated by removal of the crista from the medium with forceps. The tissue was briefly (approx. $5 \mathrm{sec}$ ) rinsed in Ringer's solution, dissolved in $50 \mu \mathrm{l}$ of $1 \mathrm{~N}$ $\mathrm{NaOH}$, neutralized with acetic acid, and counted.

Materials. Tobramycin, gentamicin and netilmicin were from the Schering Co. (Bloomfield, NJ), and neomycin from Pharma-Tek (Huntington, NY). All other chemicals were obtained from the Sigma Chemical Co. (St. Louis, MO).

Data analysis. The bound (CB) and free (CF) concentrations of gentamicin were fitted to a protein binding model which incorporates a single Langmuir term plus a constant:

$$
\mathrm{CB}=n_{1}+\frac{n_{2} * \mathrm{CF}}{K_{D_{2}}+\mathrm{CF}}
$$

$n_{1}$ represents the binding capacity for the high-affinity, low-capacity binding site; $n_{2}$ and $K_{D 2}$ represent the binding capacity and dissociation constant, respectively, for the low-affinity, high-capacity binding site. Equation 1 is derived from a binding model which incorporates two Langmuir terms:

$$
\mathrm{CB}=\frac{n_{1} * \mathrm{CF}}{K_{D 1}+\mathrm{CF}}+\frac{n_{2} * \mathrm{CF}}{K_{L 2}+\mathrm{CF}}
$$

$n_{1}, n_{2}$, and $K_{D 2}$ are the same as described previously; $K_{D 1}$ is the dissociation constant for the high-affinity, low-capacity binding site. When the free concentrations of gentamicin are much larger than the high-affinity dissociation constant $\left(\mathrm{CF}>>K_{D 1}\right)$, Equation 2 simplifies to Equation 1.

The binding parameters in Equations 1 and 2 were estimated by nonlinear regression using the MK MODEL program of the PROPHET computer system $[11,12]$ and a weighting function $1 / \mathrm{Y}^{2}$. Other models were tested (i.e, single Langmuir, single Langmuir plus linear term), but these did not fit the data well, as judged by their log-likelihood estimates $[12,13]$. The quality of the fits was further determined by evaluating standard error of the parameters, multiple- $r^{2}$ of the regression line, and by visual inspection of the residuals.

Other statistical procedures were Student's $t$-test and ANOVA, as mentioned in the legends of the respective data.

\section{RESULTS}

Preliminary experiments were designed to discriminate between intracellular drug uptake and drug either externally bound to the crista membrane or trapped within extracellular spaces. At the end of the incubation the medium was replaced with medium containing unlabeled drug at a 1000-fold higher concentration to displace all but the intracellular gentamicin. This post-incubation "wash" (Fig. 1) caused a rapid loss of about $40 \%$ of the radioactivity from the crista followed by a period of a stable radioactive content for at least $15 \mathrm{~min}$. This stable radioactivity was defined as the intracellular drug content. All subsequent assays included a 5-min postincubation prior to filtration.

Possible trapping in extracellular spaces was additionally tested with radiolabeled mannitol, a marker which is not transported into cells. Under analogous conditions to the gentamicin assay (i.e. inclusion of a post-incubation "wash"), radioactivity in the crista did not exceed background counts significantly.

Incubations with an excess of native gentamicin $(10 \mathrm{mM})$ displaced all ${ }^{125} \mathrm{I}$-labeled gentamicin and reduced the bound radioactivity to background, indicating that the radioactive gentamicin used in these experiments had the same binding characteristics as the native drug.

Gentamicin uptake was essentially linear at early times (Fig. 2). Uptake at $30 \mathrm{~min}$ was consistently high, while an apparent loss of drug at $60 \mathrm{~min}$ was seen in two experiments. Therefore, a 30-min time point was chosen for subsequent incubations. The uptake was temperature dependent (Fig. 3) and reduced by $81 \%$ at $0^{\circ}$, indicating an active transport system. In contrast, uptake of butanol, which enters cells by diffusion only, was independent of temperature $\left(2650 \pm 1300 \mathrm{cpm}\right.$ at $37^{\circ}$ versus $2500 \pm$ $1100 \mathrm{cpm}$ at $\left.0^{\circ}\right)$.

The dose-dependency of the uptake (Fig. 4) was analyzed for the affinity and capacity of the transport system. Table 1 lists the parameter estimates ( \pm SE) by the two binding models. Both analyses clearly demonstrate that two classes of binding sites are needed to describe the uptake of gentamicin. Each of the parameters derived by Equation 1 was well characterized, as evidenced by the small coefficients of variation $(\mathrm{CV}<16 \%)$. The correlation between the observed data and the computer-generated regression curve was excellent. A good fit was also achieved with the binding model described by Equation 2 (Fig. 4, insert). It should be noted that the estimates by Equations 1 and 2 are within $10 \%$ of each other for $n_{2}$ and $K_{D 2}$, and within $25 \%$ of each other for $n_{1}$. Less confidence can be placed on the numerical value of $K_{D 1}$ due to the large variability 


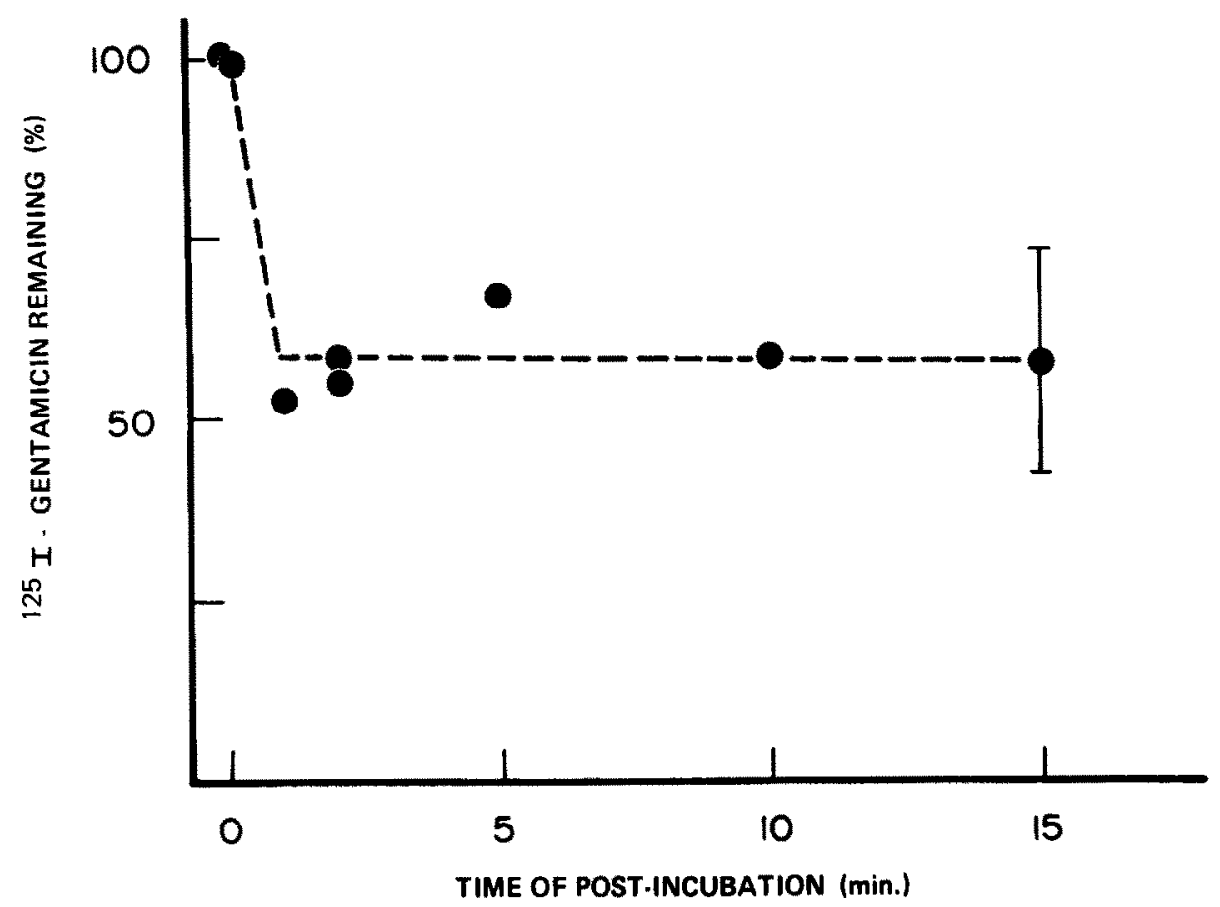

Fig. 1. Effect of post-incubation on $\left[{ }^{125} \mathrm{I}\right]$ gentamicin content of cristae. Cristae ampullares were incubated with $1 \mu \mathrm{M}{ }^{125} \mathrm{I}$-labeled gentamicin for $30 \mathrm{~min}$ as described in Materials and Methods and were then transferred to a post-incubation medium containing $1 \mathrm{mM}$ unlabeled gentamicin. Points are from individual cristae except at 0 time $(N=2)$ and 15 min $(N=3$; means $\pm S D)$.

surrounding it $(\mathrm{CV}>200 \%)$. The inability of the regression program to characterize this constant equally well as the others may be based on the fact that experimental data points below $K_{D 1}$ could not be obtained. This restraint was placed on the experiments by the specific radioactivity of the $\left[{ }^{125}\right.$ I] gentamicin.

The dose-dependency study also showed that

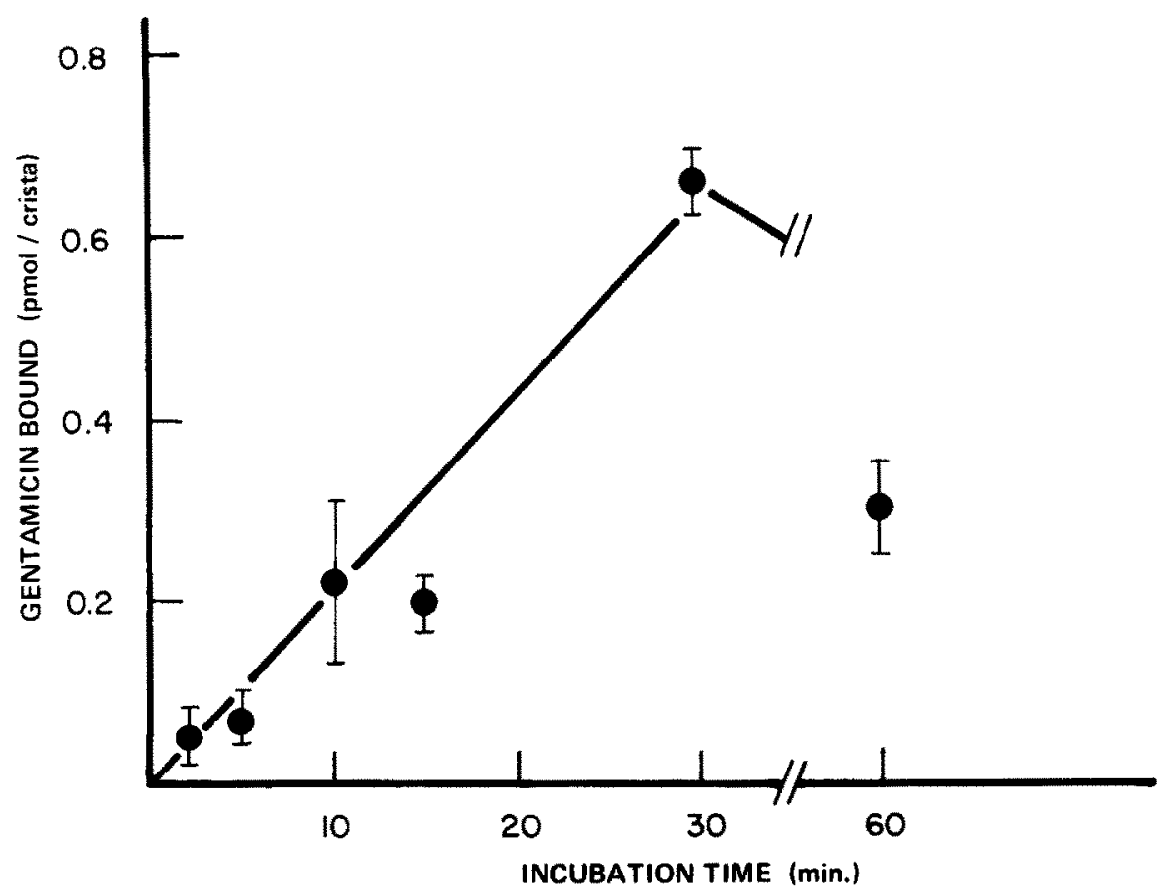

Fig. 2. Time-coursc of gentamicin uptake. Cristae ampullares were incubated with $1 \mu \mathrm{M}$ gentamicin as described in Materials and Methods. Numbers are means \pm SD from three experiments. 


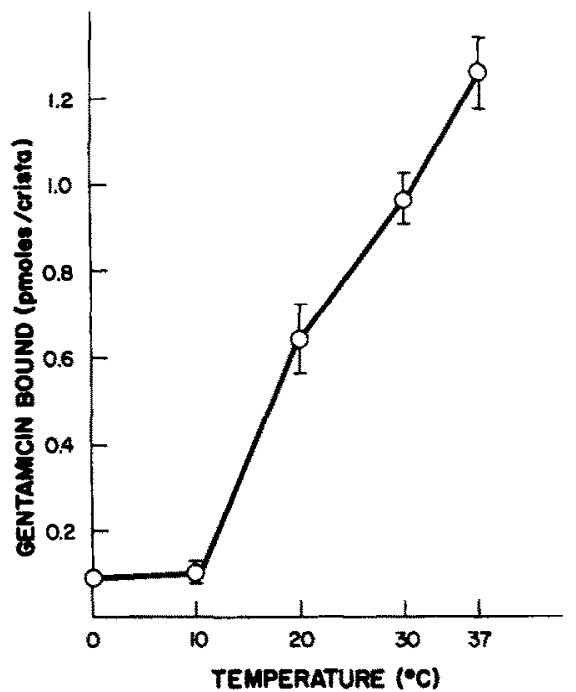

Fig. 3. Effect of temperature on gentamicin uptake. Cristae ampullares were incubated for 30 min with $1 \mu \mathrm{M}$ gentamicin at the temperatures indicated. Before the start of the incubation, cristae had been equilibrated to the respective temperatures for $5 \mathrm{~min}$. Numbers are means $\pm \mathrm{SD}$ of triplicate determinations.

gentamicin concentrations in the tissue (average wet weight of a crista, $40 \mu \mathrm{g}$ ) always exceeded those in the medium. The tissue to medium ratio was highest (18.6) at the lowest drug concentration in the medium and declined to 1.4 at $100 \mu \mathrm{M}$ gentamicin in the medium.
Gentamicin uptake was significantly inhibited by other aminoglycosides and polyamines (Table 2). Neomycin, tobramycin and netilmicin inhibited gentamicin uptake in a dose-dependent manner in the order netilmicin $\geq$ neomycin $>$ tobramycin, with $80-90 \%$ inhibition at a $100 \mu \mathrm{M}$ concentration of each drug. Spermine was the most efficient competitor among the polyamines followed by spermidine and putrescine. Conversely, gentamicin inhibited putrescine uptake (Table 3). Neither glucosamine nor the basic amino acids asparagine and lysine affected gentamicin uptake, whereas putrescine uptake was inhibited significantly by lysine $(60 \%$ at $1 \mathrm{mM})$ and asparagine $(44 \%$ at $1 \mathrm{mM})$. In addition to the compounds mentioned, the catecholamines epinephrine and norepinephrine were tested and found to have no effect on gentamicin uptake at $10 \mu \mathrm{M}$.

The addition of metabolic blockers to the incubations caused a partial inhibition of gentamicin transport (Table 4). Ouabain reduced uptake by $24 \%$, whereas iodoacetate and 2,4-dinitrophenol (DNP) were less effective. A potential transport inhibitor, the sulfhydryl group blocker $p$-chloromercuribenzoic sulfonate (pCMBS), and colchicine, which blocks pinocytosis, did not affect gentamicin uptake significantly. The effects of these agents on putrescine transport were not significantly different from their effects on gentamicin transport. In contrast, a brief comparison with the high-affinity uptake of GABA showed significant differences in the response of this system to low temperature (completely inhibited by ice) and metabolic blockers (inhibited $65 \%$ by DNP, and $77 \%$ by ouabain).

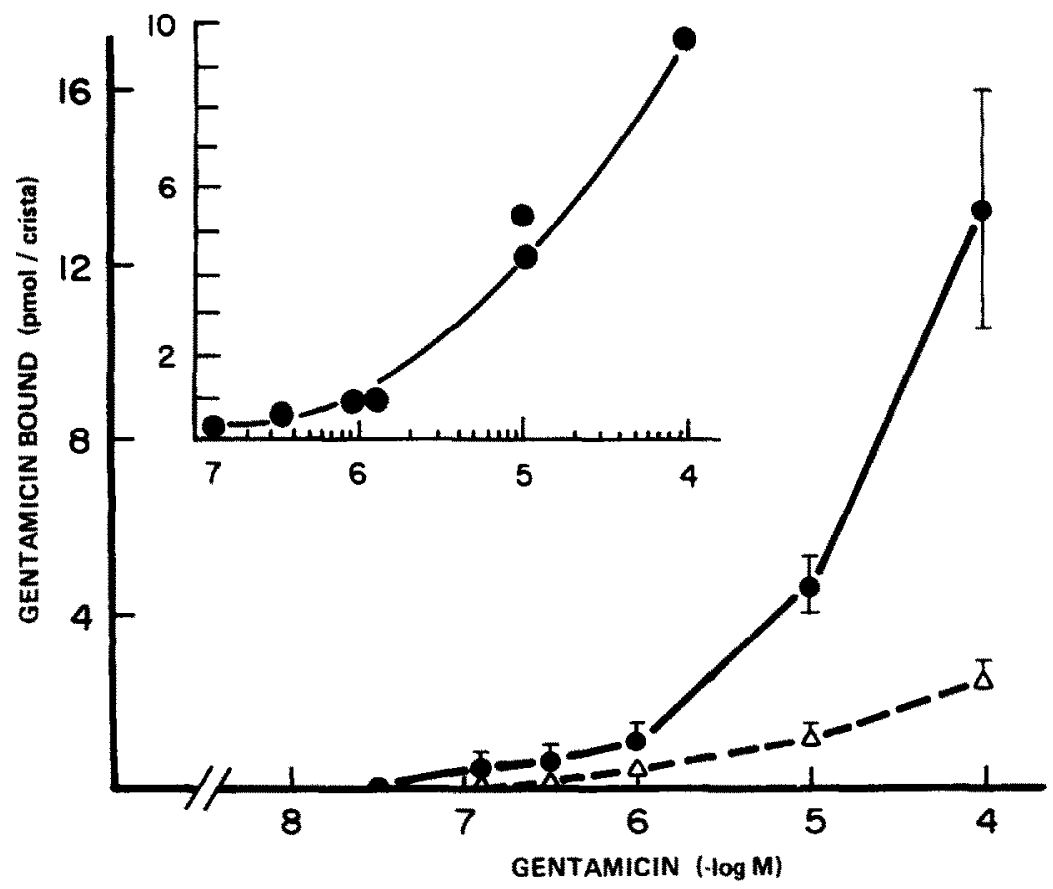

Fig. 4. Dose-dependency of gentamicin uptake. Cristae ampullares were incubated for 30 min with the indicated concentrations of gentamicin at $37^{\circ}(-)$ or $0^{\circ}(\Delta-\Delta)$. Numbers are means $+\mathrm{SD}$ of triplicate determinations. Insert: Computer-fitted curve (by Equation 2; see Materials and Methods) to the experimental data of the temperature-sensitive uptake, i.e. values of the incubation at $0^{\circ}$ subtracted from the values of uptake at $37^{\circ}$. 
Table 1. Parameters of gentamicin uptake

\begin{tabular}{llllllr}
\hline $\begin{array}{l}\text { Method of } \\
\text { calculation }\end{array}$ & \multicolumn{2}{c}{$\begin{array}{c}n_{1} \\
\text { (pmoles/crista) }\end{array}$} & $n_{21}$ & $K_{D 2}$ & $r^{2^{*}}$ & $\log \mathrm{L}+$ \\
\hline Equation 1 & $0.18 \pm 0.03 \ddagger$ & $11.0 \pm 1.1$ & & $15,011 \pm 2,382$ & 0.9998 & -5.27 \\
Equation 2 & $0.24 \pm 0.12$ & $11.2 \pm 1.3$ & $39.2 \pm 79.4$ & $16,313 \pm 3,979$ & 0.9998 & -4.87 \\
\hline
\end{tabular}

* Multiple $-r^{2}$ of the regression line.

$\dagger$ Log-likelihood estimate [12,13].

$\ddagger$ Numbers are parameter estimates \pm SE of the regression parameters.

\section{DISCUSSION}

In an intact organ such as the crista ampullaris, a drug may occupy several distinct locations: extracellular spaces, extracellular binding sites and intracellular compartments. Before mechanisms of uptake can be characterized the extracellular contributions need to be assessed and, if possible, eliminated. This seems particularly important in a study of gentamicin, where extracellular drug binding may be a quantitatively major factor due to the high affinity of aminoglycosides for negatively charged surface components. Our experimental design defines intracellular gentamicin as being sequestered in a stable, not easily exchangeable pool. This pool is experimentally isolated by a post-incubation with excess unlabeled drug which causes a rapid displacement of about $50 \%$ of the initially present radioactivity. This rapid displacement is indicative of superficial binding sites and the stable radioactivity

Table 2. Effect of competitors on gentamicin uptake

\begin{tabular}{lcccc}
\hline & \multicolumn{5}{c}{ Uptake (\% of control) } \\
\cline { 2 - 5 } Competitor & \multicolumn{4}{c}{ Concentration of competitor } \\
& $1 \mu \mathrm{M}$ & $10 \mu \mathrm{M}$ & $100 \mu \mathrm{M}$ & $1 \mathrm{mM}$ \\
\hline Netilmicin* & $53 \pm 35$ & $36 \pm 8$ & $16 \pm 4$ & \\
Neomycin & $71 \pm 12$ & $52 \pm 14$ & $10 \pm 1$ & \\
Tobramycin & $92 \pm 13$ & $71 \pm 9$ & $18 \pm 8$ & \\
Putrescine $\dagger$ & & $72 \pm 21$ & $72 \pm 11$ & $75 \pm 5$ \\
Spermidine & & $69 \pm 21$ & $59 \pm 11$ & $24 \pm 14$ \\
Spermine & & $89 \pm 9$ & $22 \pm 4$ & \\
Asparagine $\dagger$ & $110 \pm 40$ & & $90 \pm 23$ & $143 \pm 15$ \\
Lysine & $124 \pm 8$ & & $73 \pm 2$ & $193 \pm 52$ \\
Glucosamine & $90 \pm 17$ & & $95 \pm 27$ & $120 \pm 1$
\end{tabular}

Uptake was analyzed at $1 \mu \mathrm{M}$ gentamicin as described in Materials and Methods. Numbers are means \pm SD of at least triplicate determinations. Control value for gentamicin uptake was $1.05 \pm 0.31$ pmoles $/$ crista $(N=75)$.

* Treatment differs from control, $P<0.01$ for each aminoglycoside; differences between drugs: netilmicin vs tobramycin, $\mathrm{P}<0.01$; netilmicin vs neomycin, $\mathrm{P}=0.27$; neomycin vs tobramycin, $P=0.025$ (two-way ANOVA).

$\dagger$ Treatment differs from control, $\mathrm{P}<0.01$ for each polyamine; differences between polyamines (at $100 \mu \mathrm{M}$ and $1 \mathrm{mM}$ ): putrescine vs spermidine, $\mathbf{P}<0.01$, putrescine vs sperminc, $\mathrm{P}<0.01$; spermidine vs spermine, $\mathrm{P}<0.069$ (ANOVA).

$\ddagger$ Treatment with asparagine, lysine, glucosamine, not different from control. signifies a deeper compartment. The absence of extracellular trapping in the crista was also confirmed in incubations with mannitol, a marker of extracellular spaces. After analogous procedures, no radioactivity was incorporated into the cristae.

In contrast to the retention of radiolabeled gentamicin in the presence of an excess of unlabeled drug after the incubation, all radioactivity was eliminated when such an excess of unlabeled gentamicin was added to the initial incubation. This displacement demonstrated that non-specific binding of $\left.{ }^{125} \mathrm{I}\right]$ gentamicin did not occur as all binding sites were accessible to the native drug. This documentation of ${ }^{125}$ I-labeled gentamicin as a valid substitute for gentamicin is in agreement with Humes et al. [14] who studied the renal binding of this compound.

Several lines of evidence point to an energy-dependent process underlying or at least contributing to the uptake of gentamicin in the crista. First, the uptake occurred against a concentration gradient yielding a tissue to medium ratio of 1.4 to 18.6 , depending on the drug concentration in the medium. This was remarkably similar to observations in the developing inner ear where tissue levels of gentamicin exceed those in the medium up to 17 -fold [9]. Likewise, kidney slices have been reported to transport gentamicin actively to a 2 - to 5-fold excess in the tissue. Second, the uptake was temperaturedependent; $80 \%$ could be eliminated by cooling the crista to 0 or $10^{\circ}$. The remaining $20 \%$ may represent a fraction of the drug entering by diffusion. Diffusion is unaffected by temperature as seen in the temperature-independence of the tissue levels of butanol, which enters cells exclusively by this process.

Table 3. Effects of competitors on putrescine uptake

\begin{tabular}{lcc}
\hline & \multicolumn{2}{c}{ Uptake (\% of control) } \\
\cline { 2 - 3 } Competitor & $\begin{array}{c}\text { Concentration of competitor } \\
100 \mu \mathrm{M}\end{array}$ & $1 \mathrm{mM}$ \\
\hline Gentamicin & $70 \pm 24$ & $56 \pm 15^{*}$ \\
Asparagine & $80 \pm 22$ & $56 \pm 22 \dagger$ \\
Lysine & $51 \pm 6$ & $40 \pm 14 \dagger$ \\
\hline
\end{tabular}

Uptake was analyzed at $1 \mu \mathrm{M}$ putrescine as described in Materials and methods. Numbers are means \pm SD of at least triplicate determinations. Control value for putrescine uptake was $4.01 \pm 1.7$ pmoles $/$ crista $(\mathrm{N}=30)$.

* Treatment differs from control, $\mathrm{P}=0.02$.

$\dagger$ Treatment differs from control, $\mathrm{P}<0.01$. 
Table 4. Effects of inhibitors on uptake

\begin{tabular}{lccc}
\hline & \multicolumn{3}{c}{ Uptake as (\% of control) } \\
\cline { 2 - 4 } Inhibitor & Gentamicin & Putrescine & GABA \\
\hline Ice & $19 \pm 12^{*}$ & $5 \pm 3^{*}$ & $0 \pm 0^{*}, \dagger$ \\
Ouabain & $76 \pm 19^{*}$ & $56 \pm 16^{*}$ & $23 \pm 16^{*} *$ \\
DNP & $87 \pm 10$ & $73 \pm 29$ & $35 \pm 11^{*}, \neq$ \\
Iodoacetate & $88 \pm 14$ & $\mathrm{ND}$ & $\mathrm{ND}$ \\
pCMBS & $138 \pm 30$ & $74 \pm 37$ & $\mathrm{ND}$ \\
Colchicine & $82 \pm 24$ & $73 \pm 27$ & $\mathrm{ND}$ \\
\hline
\end{tabular}

Cristae were incubated with the inhibitors and radiolabeled gentamicin, putrescine or GABA as described in Materials and Methods. Inhibitor concentrations were DNP, $1 \mathrm{mM}$; ouabain, $1 \mathrm{mM}$; iodoacetate, $10 \mathrm{mM}$; pCMBS (para-chloromercuribenzoic sulfonate), $50 \mu \mathrm{M}$; and colchicine, $2 \mu \mathrm{M}$. "Ice" indicates a 30 -min incubation on ice. Numbers are means $\pm S D$ of three to nine experiments per inhibitor, each in triplicate. ND $=$ not determined. Control uptake values: gentamicin, $1.05 \pm 0.31$ pmoles $/$ crista $(N=$ $75)$; putriscine, $4.01 \pm 1.7$ pmoles/crista $(N=30)$; and GABA, $0.196 \pm 0.08$ pmoles $/$ crista $(\mathrm{N}=10)$.

* Differs from control, $\mathrm{P}<0.01$ (paired $t$-test).

$\dagger$ Differs from effect on gentamicin uptake, $P=0.028$.

$¥$ Differs from effect on gentamicin uptake, $P<0.01$.

Gentamicin uptake was also partially susceptible to metabolic inhibitors. In the absence of an independent measure of ATP levels in the tissue, the significance of this partial block remains uncertain. It is possible that these agents require longer incubation times to exert their actions. For example, iodoacetate effectively blocks renal gentamicin uptake after $60 \mathrm{~min}$ but is ineffective at short incubation times [4].

The active transport system, defined as the difference between uptake at $37^{\circ}$ and $0^{\circ}$, had at least two components. The first was a high-affinity system with a remarkably low $K_{D}=39 \mathrm{nM}$ and a capacity of 0.2 pmole/crista. The second site was clearly evident in the analysis although no saturation of uptake was achieved under the experimental conditions. Both the affinity and capacity of the second site were several orders of magnitude different from those of the first site: $K_{D}=16 \mu \mathrm{M}, n=11$ pmoles/crista.

Gentamicin appears to enter the crista, at least in part, by the polyamine transport system. The polyamines inhibited gentamicin uptake in the order of their increasing positive charge, spermine $>$ spermidine $>$ putrescine, an observation similarly made in the kidney [15]. Gentamicin, in turn, competed with putrescine uptake. The direct comparison of gentamicin and putrescine uptake did not show any significant difference in the effect of potential inhibitors on these two systems. In contrast, the uptake for the amino acid GABA reacted significantly differently to such treatment. The responses of gentamicin and putrescine uptakes to basic amino acids and glucosamine, however, were not parallel. This discrepancy could be resolved by the assumption that putrescine, but not gentamicin, could also be transported by the basic amino acid transport system in the crista ampullaris. Then part of the total polyamine but not gentamicin uptake would be susceptible to inhibition by amino acids.
Glucosamine (2-amino-D-glucose), an inhibitor of glucose uptake, was included in this study because of suggestions that aminoglycosides may exert their toxic actions through interference with glucose transport [16]. Gentamicin uptake was unaffected by glucosamine, confirming our previous findings that inhibition of glucose uptake and utilization is not a primary mechanism of aminoglycoside toxicity $[17,18]$,

A number of parallels are evident between the renal uptake system and that in the crista. As pointed out above, drug accumulation, temperature dependence, and sensitivity to metabolic blockers are similar. The parameters of uptake by the second site in the crista are well in the range of those reported for uptake in renal brush border membranes $[19,20]$. Furthermore, gentamicin uptake in both tissues is competitive with other aminoglycosides and polyamines, but not with basic amino acids $[4,5,15,21]$. These parallels suggest that a similar uptake system is an important element in the toxicity of aminoglycosides to both the kidney and the inner ear.

In assessing the contribution of the active transport to the toxicity of the aminoglycosides, two aspects should be noted. The first is the very high affinity of the uptake system $\left(K_{D}=39 \mathrm{nM}\right)$ which should provide for an efficient tissue entry of these drugs even at low serum concentrations. The second point is that the order of affinity for the uptake system, netilmicin = gentamicin $\geqslant$ neomycin $>$ tobramycin is not the order of the vestibulo- or cochleotoxicity of these drugs. Thus, while the active uptake may aid in achieving toxic drug concentrations in the affected tissues and thus represent an integral part of the ototoxic mechanism, it is not the step that determines the extent of drug toxicity in that tissue. Those controls may lie-as we have demonstrated before-in specific drug interactions with other intracellular components such as phosphatidylinositol $4,5-$ bisphosphate [22].

Acknowledgements-The authors wish to acknowledge the help of Ted Diamond with the statistical analyses. This work was supported by NIH Research Grant NS-13792.

\section{REFERENCES}

1. R. E. Hancock, J. Antimicrob. Chemother. 8, 249 (1981).

2. W. M. Kluwe and J. B. Hook, Toxic. appl. Pharmac. 45, 531 (1978).

3. P. Pastoriza-Munoz, R. L. Bowman and G. J. Kaloyanides, Kidney Int. 16, 440 (1979).

4. W. Bennett, C. Plamp, C. Elliot, R. Parker and G. Porter, J. Lab. clin. Med. 22, 156 (1981).

5. J. Lipsky, L. Cheng, B. Sacktor and P. Lietman, $J$. Pharmac. exp. Ther. 215, 390 (1980).

6. P. Tran Ba Huy, P. Bernard and J. Schacht, J. clin. Invest. 77, 1492 (1986).

7. A. Takada and J. Schacht, Hear. Res, 8, 179 (1982).

8. A. Takada, S. Bledsoe and J. Schacht, Hear. Res. 19, 245 (1985).

9. J. Schacht and T. Van De Water, Biochem. Pharmac. 35,2843 (1986).

10. M. Anniko, D. Bagger-Sjöbäck, J. Wersäll and J. Schacht, Res. Commun. Chem. Path. Pharmac. 37, 333 (1982).

11. W. F, Raub, Fedn. Proc. 33, 2390 (1976). 
12. N. H. G. Holford, in Prophet Public Procedures Notebook (Ed. H. M. Perry), p. 85. Bolt Beranek \& Newman, Cambridge, MA (1982).

13. G. Schwartz, Ann. Statist. 6, 461 (1978).

14. H. Humes, M. Sastrasinh and J. Weinberg, J. clin. Invest. 73, 134 (1984).

15. C. Josepovitz, E. Monoz, D. Timmerman, M. Scott, S. Feldman and G. Kaloyanides, J. Pharmac. exp. Ther. 223, 314 (1982).

16. J. Garcia-Quiroga, C. H. Norris, L. Glade, G. M. Bryant, M. Tachibana and P. Guth, Res. Commun. Path. Pharm. 22, 535 (1978).
17. M. Tachibana, W. Jung, K. Schindler and J. Schacht, Archs Oto-rhino-laryngol. 230, 103 (1981).

18. A. Takada, B. Canlon and J. Schacht, Res. Commun. Chem. Path. Pharmac, 42, 203 (1983).

19. M. Sastrasinh, T. Knauss, J. Weinberg and H. Humes, J. Pharmac. exp. Ther. 222, 350 (1982).

20. M. Just and E. Habermann, Naunyn.Schmiedeberg's Archs Pharmac. 300, 67 (1977).

21. J. Höltje, Eur. J. Biochem. 86, 345 (1978).

22. J. Schacht, Hear. Res. 22, 297 (1986). 